\title{
Study of the magnitude of diabetes and its associated risk factors among the tuberculosis patients of Morang, Eastern Nepal
}

\author{
Babita Sharma* (D), Vijay Kumar Khanal, Nilambar Jha, Prajjwal Pyakurel and Gyanu Nepal Gurung
}

\begin{abstract}
Background: WHO addresses the infectious disease like Tuberculosis, and non- communicable disease like Diabetes among the top 10 causes of death worldwide, which collectively leads to increasing mortality and premature death especially in developing countries. Hence, the present study aims to assess the prevalence of diabetes and its associated risk factors among the tuberculosis patient of Morang, Eastern Nepal.

Methods: A cross-sectional study was carried out among the 320 respondents undergoing tuberculosis treatment of Morang district. Respondents from eight randomly selected DOTS centers were selected purposively. The Fasting Blood Sugar and 2-h Post-Prandial Blood Sugar were assessed in the laboratory of respective DOTS center by the glucose oxidase method. An interview for socio-demographic and other variables was conducted using a pretested semi-structured questionnaire based on WHO-STEP Instrument for chronic disease and excerpt from DASS-21 was used for the variable stress.

Results: The prevalence of diabetes, pre-diabetic and glucose intolerance among tuberculosis patient was 11.9, 17.2, and $17.8 \%$ respectively. Additionally, the univariate analysis reported, user of tobacco products, current alcohol consumers, family history of diabetes and stress level, to have positive association with diabetes, while the multivariate analysis reported, the current alcohol consumer as the significant predictor of diabetes among the tuberculosis patient.

Conclusion: A significant portion of the respondents were diabetic, impaired glucose tolerance and pre-diabetic, which supports the fact of diabetes being comorbid with tuberculosis. Hence, it shifts the focus on the bidirectional screening of tuberculosis and diabetes.
\end{abstract}

Keywords: Diabetes, Tuberculosis, Co-morbidity, Prevalence, Eastern Nepal

\section{Background}

Diabetes mellitus (DM) is a serious lifelong condition, which occurs when the pancreas does not produce enough insulin, or when the body cannot effectively use the insulin it produces leading to hyperglycemia [1].

In contrast to $\mathrm{DM}$, tuberculosis (TB), is an infectious bacterial disease caused by Mycobacterium tuberculosis, which most commonly affects the lungs. People infected with TB bacteria have a $5-15 \%$ lifetime risk of falling ill

* Correspondence: babeetasharma1@gmail.com

School of Public Health and Community Medicine (SPHCM), B. P. Koirala Institute of Health Sciences (BPKIHS), Dharan, Nepal with TB. However, persons with compromised immune systems, such as people living with HIV, malnutrition or diabetes, or people who use tobacco, have a much higher risk of falling ill [1].

WHO addresses the infectious disease like TB, and NCD like DM among the top 10 causes of death worldwide [1]. DM has been increasing in prevalence year after year, assumed to target mainly LMIC rather than highincome countries. In contrast to DM, TB occurs in every part of the world. However, the epidemic growth of DM especially occurs in developing countries, where TB is highly endemic, leading to increased mortality $[2,3]$.

(c) The Author(s). 2019 Open Access This article is distributed under the terms of the Creative Commons Attribution 4.0 International License (http://creativecommons.org/licenses/by/4.0/), which permits unrestricted use, distribution, and reproduction in any medium, provided you give appropriate credit to the original author(s) and the source, provide a link to the Creative Commons license, and indicate if changes were made. The Creative Commons Public Domain Dedication waiver (http://creativecommons.org/publicdomain/zero/1.0/) applies to the data made available in this article, unless otherwise stated. 
Importantly, patients with $\mathrm{TB}$ who have $\mathrm{DM}$ also have worse TB treatment than those who do not have DM, which includes delayed conversion from positive to negative sputum cultures, higher risk of death during TB treatment and a higher risk of relapse after successfully completed treatment [2, 3].

Despite, TB in Nepal remains to be a major public health problem, minimal studies have been carried out regarding DM-TB comorbidity, which reveals the considerable prevalence of DM among TB patients (9.1\%) [4]. Moreover, the SEAR experiences more unsettling problem of MDR-TB which worsens through comorbidities such as HIV and DM with TB, and jeopardizes the global objectives of ending TB by 2030 and eliminating TB by $2050[2,5]$.

The aim of the study is to determine the prevalence of diabetes and its associated risk factors among tuberculosis patients. The findings from the study will support the government for the collaborative framework of bidirectional screening of DM-TB comorbidity leading to early detection and treatment of both the disease simultaneously, decreasing the national prevalence of both DM and TB, and promoting the mental health and wellbeing of the people.

\section{Methods}

A cross-sectional study of 6-month duration (1st September 2018 to 28th February 2019) was conducted at DOTS centers with laboratory facility of Morang, Eastern Nepal. Morang district consisted of 71 DOTS center including 20 with laboratory facility. Out of those 20, 8 DOTS center were randomly selected using lottery method.

\section{Sample size}

All the TB patients aged 15 years and above who were registered under the National Tuberculosis Control Program and on treatment during the study period with completed intensive phase (2 months) treatment were included in the study. A sample size of 320 was calculated using a formula $n=z^{2} p q / e^{2}$ considering the prevalence from the study done in 2014 by Raghuraman et all in urban Puducherry and non-response rate of $10 \%$.

\section{Data collection}

A pretested semi-structured questionnaire based on WHO-STEPS instrument was used to collect the information related socio-demographic characteristics and behavioral risk factors [14]. Global Physical Activity Questionnaire (GPAQ) developed by WHO was used for assessing physical activity [14]. And, an excerpt from DASS-21 was used for the variable stress [15]. A face-toface interview was taken by the researcher herself. Floor weighing scale for weight, and Linen tape for height, hip and waist circumference measurement were used for the anthropometric measurement. Fasting plasma glucose level and 2-h post-prandial glucose were estimated in the laboratory of the respective DOTS center by glucoseoxidase method, which used $2 \mathrm{ml}$ of patient's blood sample collected for each, by venipuncture. The blood sample was collected by the trained laboratory technician of the respective DOTS center.

On the first day, the purpose of the study was explained to the patient and participant information sheet written in Nepali (local language) was provided and informed written consent was obtained. The participants were asked to come the next day after overnight fasting for the test of fasting blood glucose (FBG) levels and were asked again to report after $2 \mathrm{~h}$ of having lunch for the 2-h post-prandial (PP) glucose test. The patients who had already been diagnosed for diabetes, details of the time of diagnosis and treatment taken was collected by asking medicine slip/ patient's cards/ diagnosis slip to ensure the confirmation of the self-reported DM.

\section{Data analysis}

Collected data were entered in Microsoft Excel 2016 and converted in Statistical Package for Social Science 11.5 version for statistical analysis. Descriptive analysis was done in the form of frequencies, percentages, mean, median and standard deviation. For inferential statistics, Chi-square test was applied to test the association between DM and others selected risk factors at 95\% CI and level of significance as $P=0.05$ for categorical data, while "students t-test" was used for continuous data. Variables with $P$ value $<0.20$ and with expected cell count not less than 5 were subjected to multivariate analysis (Binary Logistic Regression with category).

\section{Ethical consideration}

Approval for this study was obtained following proposal review from the Institutional Review Committee (IRC) of BPKIHS (Code No: IRC/1265/018). Informed written consent was taken from respondents. Consent for publication has been taken from the author, co-authors and the respondents.

\section{Results}

Socio-demographic and socio-economic characteristics of the respondents

The study included 320 respondents, selected from 8 randomly chosen different DOTS centers of Morang, Eastern Nepal. The mean age of the respondents was 41.5 years with an abundance of male (70.0\%). More respondent $(70.9 \%)$ were Hindu by religion, and many (42.5\%) were Janajati by their ethnicity. Likewise, many $(62.2 \%)$ were currently married and had nuclear family (64.1\%). 
Tuberculosis and diabetic status of the respondents In this study, 38 (11.9\%) respondents were found to have diabetes which includes 19 (50.0\%) self-reported cases of diabetes. The remaining 19 (50.0\%) were the new case diagnosed during the time of the study. Among those 19 self-reported diabetes cases, the study also reflected 9 $(47.4 \%)$ to have a known history of diabetes prior to the tuberculosis treatment, with a median duration of five months. Despite, the low prevalence of diabetes (11.9\%) in our study, there still exist a considerable portion $(17.8 \%)$ of the respondents with impaired glucose and impaired fasting (17.8\%). Table 1.

\section{Behavioral characteristics}

Among 320 respondents, 58 (18.1\%) was a current user of tobacco products; $56(96.6 \%)$ and $2(3.4 \%)$ as daily and non-daily users of tobacco products respectively. This study finding also suggests that 179 (55.9\%) and 83 (25.9\%) were former user and never used tobacco products, respectively as categorized as non-user group in this study. Among those current users of tobacco products, many respondents $35(60.3 \%)$ preferred smokeless tobacco. Nevertheless, further adjusting for different variables, the current user of tobacco products turns out to

Table 1 Tuberculosis and Diabetic Status of the respondents

\begin{tabular}{lcc}
\hline Characteristics & Frequency $(n=320)$ & Percent (\%) \\
\hline TB Type & 126 & $39.4 \%$ \\
Sputum Positive & 142 & $44.4 \%$ \\
Sputum Negative & 52 & $16.2 \%$ \\
Extra Pulmonary & & \\
TB Treatment Type & 285 & $89.1 \%$ \\
New Case & 35 & $10.9 \%$ \\
Relapse Case & \\
Diabetes-Tuberculosis Comorbidity & $88.1 \%$ \\
TB Only & 282 & $11.9 \%$ \\
TB-DM & 38 & \\
Impaired Glucose Tolerance (IGT) & $17.8 \%$ \\
Yes & 57 & $82.2 \%$ \\
No & 263 & \\
Impaired Fasting Glucose (IFG) & & \\
Yes (Pre-Diabetic) & 55 & $17.2 \%$ \\
No & 265 & $82.8 \%$ \\
Diabetic Status & & \\
Yes (Self-reported) & 19 & $5.9 \%$ \\
No & $47.4 \%$ \\
Don't Know & 254 & \\
History of DM before TB Treatment $(n=19)$ & \\
Yes & 9 & \\
No & $10.7 \%$ \\
\hline
\end{tabular}

have a positive association with an odd of 2.247 times $(\mathrm{OR}=2.247,0.728-11.984)$.

However, the study findings reflect a small portion 14 (4.3\%) of the respondents who currently drink the alcohol and 126 (39.4\%) of each had drunk alcohol in 12 months and lifetime abstainer. Despite, a small portion (12.0\%) of respondents constituted for who currently drinks alcohol $(\mathrm{OR}=16.167,4.262-61.322)$, after adjusting for other variables, they were found to have a very strong positive association for the development of diabetes with an odd of 12.307 times ( $\mathrm{OR}=12.307,2.856-57.067)$.

The family history is the irreversible risk factor to diabetes is positively associated with diabetes, and the odds of getting diabetes is 2.463 times $(\mathrm{OR}=2.463,0.638-10.024)$.

Interestingly, in this study, neither the mean servings of fruits and vegetables nor the physical activity showed an independent association with diabetes. Tables 2 and 4.

\section{Physical measures}

The study reported abdominal obesity in more than half $(55.2 \%)$ and one third $(33.5 \%)$ of female and male, respectively and collectively, $40.0 \%$ of the total respondents in this study to have abdominal obesity. However, the bivariate analysis did not reflect an independent association of waist-to-hip (WHR) with diabetes.

With regard to the blood pressure of the respondents, the study reported a considerable portion $(27.2 \%)$ of the respondents to be pre-hypertensive. However, the bivariate analysis did not reflect an independent association of blood pressure range with diabetes. Tables 3 and 4.

\section{Stress level}

The present study finding reports two-third (63.1\%) of the respondents having severe stress, and also reflects the positive association of diabetes with increasing level of stress. The odds for mild-moderate and severe level of stress of getting diabetes was 1.930 times $(\mathrm{OR}=1.930$, $0.407-9.158)$ and 3.816 times ( $\mathrm{OR}=3.816,0.540-26.983)$, respectively compared to the normal stress level.

\section{Discussion}

\section{Tuberculosis and diabetic status of the respondents}

Out of 320 respondents selected from 8 randomly chosen different DOTS centers of Morang, Eastern Nepal, the prevalence of diabetes in the present study was found slightly higher (9.1\%) than the study conducted in 2013 in Kathmandu [4] valley and China (6.3\%) [6], however, lower enough than India [7, 8] and Pakistan [5] which reported the prevalence in the range of 20.0 to $30.0 \%$. This was justified by the considerable portion of (42.5\%) Janajati in the present study, as their culture and customs of drinking alcohol adjuvants the risk factor of diabetes among those people. 
Table 2 Behavioral Characteristics of the Respondents

\begin{tabular}{|c|c|c|c|c|c|}
\hline \multirow{2}{*}{$\begin{array}{l}\text { Characteristics } \\
\text { Use of Tobacco Product }\end{array}$} & & \multirow[t]{2}{*}{ TB only $(n=282)$} & \multirow[t]{2}{*}{ TB-DM $(n=38)$} & \multirow[t]{2}{*}{ Total $(n=320)$} & \multirow[t]{2}{*}{$P$-value } \\
\hline & & & & & \\
\hline \multirow[t]{3}{*}{ Current User $(n=58)$} & Daily & $43(97.7 \%)$ & $13(92.9 \%)$ & $56(96.6 \%)$ & \multirow[t]{3}{*}{$0.001^{* a}$} \\
\hline & Non-Daily & $1(2.3 \%)$ & $1(7.1 \%)$ & $2(3.4 \%)$ & \\
\hline & Total & $44(100.0 \%)$ & $14(100.0 \%)$ & $58(100.0 \%)$ & \\
\hline \multirow[t]{3}{*}{ Non-User $(n=262)$} & Former & $168(70.6 \%)$ & $11(45.8 \%)$ & $179(68.3 \%)$ & \multirow[t]{3}{*}{$0.013^{* a}$} \\
\hline & Never & 70 (29.4\%) & $13(54.2 \%)$ & $83(31.7 \%)$ & \\
\hline & Total & $238(100.0 \%)$ & $24(100.0 \%)$ & $262(100.0 \%)$ & \\
\hline \multicolumn{6}{|l|}{ Alcohol Consumption } \\
\hline \multirow[t]{2}{*}{ Current Drinker (in 30 days) } & Yes & $7(6.4 \%)$ & $7(53.8 \%)$ & $14(12.0 \%)$ & \multirow[t]{2}{*}{$<0.001^{* a}$} \\
\hline & No & 97 (93.3\%) & $6(46.2 \%)$ & $103(88.0 \%)$ & \\
\hline \multirow[t]{2}{*}{ Drank in past (12 months) } & Yes & $107(63.3 \%)$ & $13(52.0 \%)$ & $126(61.9 \%)$ & \multirow[t]{2}{*}{$0.277^{\mathrm{a}}$} \\
\hline & No & $62(36.7 \%)$ & $12(48.0 \%)$ & $74(38.1 \%)$ & \\
\hline \multicolumn{2}{|l|}{ Lifetime abstainer } & $113(40.1 \%)$ & $13(34.2 \%)$ & $126(39.4 \%)$ & $0.488^{\mathrm{a}}$ \\
\hline \multicolumn{2}{|c|}{ Mean serving of fruits and vegetables per day } & $2.29 \pm 0.63$ & $2.18 \pm 0.62$ & - & $0.343^{c}$ \\
\hline \multicolumn{6}{|l|}{ Physical Activity } \\
\hline \multicolumn{2}{|l|}{ Low Physical Activity } & $110(39.0 \%)$ & $13(34.2 \%)$ & $123(38.4 \%)$ & \multirow[t]{3}{*}{$0.178^{\mathrm{a}}$} \\
\hline \multicolumn{2}{|l|}{ Moderate Physical Activity } & $148(52.5 \%)$ & $18(47.4 \%)$ & $166(51.9 \%)$ & \\
\hline \multicolumn{2}{|l|}{ High Physical Activity } & $24(8.5 \%)$ & $7(18.4 \%)$ & $31(9.7 \%)$ & \\
\hline \multicolumn{6}{|l|}{ Family History of DM } \\
\hline \multicolumn{2}{|l|}{ Yes } & $71(25.2 \%)$ & $24(63.2 \%)$ & 95 (29.7\%) & \multirow[t]{2}{*}{$<0.001^{* b}$} \\
\hline \multicolumn{2}{|l|}{ No } & 211 (74.8\%) & 14 (36.8\%) & 225 (70.3\%) & \\
\hline
\end{tabular}

"**" Significant Association $(P<0.05)$

" $a$ " Pearson Chi-Square, " $b$ " Liner-by-Linear Association, "c" Independent Sample t-Test

Table 3 Association of the Physical Measurements and diabetic status of the respondents

\begin{tabular}{|c|c|c|c|c|c|}
\hline \multicolumn{2}{|c|}{ Characteristics } & TB only $(n=282)$ & TB-DM $(n=38)$ & Total $(n=320)$ & $P$-value \\
\hline \multicolumn{6}{|l|}{$\overline{\mathrm{BMI}}$} \\
\hline \multicolumn{2}{|c|}{ Underweight $(<18.5$ kg/m²) } & $146(51.8 \%)$ & $22(57.9 \%)$ & $168(52.5 \%)$ & $0.738^{\mathrm{a}}$ \\
\hline \multicolumn{2}{|c|}{ Ideal Weight $\left(18.5-22.9 \mathrm{~kg} / \mathrm{m}^{2}\right)$} & 105 (37.2\%) & $11(28.9 \% \%)$ & 116 (36.2\%) & \\
\hline \multicolumn{2}{|c|}{ Overweight ( $\geq 23$ kg/m²) } & $31(11.0 \%)$ & $5(13.2 \%)$ & $36(11.3)$ & \\
\hline \multicolumn{6}{|c|}{ Waist-to-Hip ratio } \\
\hline \multirow[t]{2}{*}{ Male } & Obesity & $65(32.7 \%)$ & $10(40.0 \%)$ & 75 (33.5\%) & $0.464^{b}$ \\
\hline & Normal & $134(67.3 \%)$ & $15(60.0 \%)$ & $149(66.5 \%)$ & \\
\hline \multirow[t]{2}{*}{ Female } & Obesity & 47 (56.6\%) & $6(46.2 \%)$ & $53(55.2 \%)$ & $0.480^{b}$ \\
\hline & Normal & $36(43.4 \%)$ & $7(53.8 \%)$ & $43(44.8 \%)$ & \\
\hline \multicolumn{6}{|c|}{ Blood Pressure } \\
\hline \multicolumn{2}{|l|}{ Normal } & $151(53.5 \%)$ & $23(60.5 \%)$ & $174(54.4 \%)$ & $0.879^{a}$ \\
\hline \multicolumn{2}{|c|}{ Pre-hypertension } & $81(28.7 \%)$ & $6(15.8 \%)$ & $87(27.2 \%)$ & \\
\hline \multicolumn{2}{|c|}{ Stage 1 Hypertension } & $47(16.7 \%)$ & $9(23.7 \%)$ & $56(17.5 \%)$ & \\
\hline \multicolumn{2}{|c|}{ Stage 2 Hypertension } & $3(1.1 \%)$ & 0 & $3(0.9 \%)$ & \\
\hline \multicolumn{2}{|c|}{ Mean Systolic Blood Pressure } & $117.23 \pm 16.86$ & $115.53 \pm 18.41$ & - & $0.562^{c}$ \\
\hline \multicolumn{2}{|c|}{ Mean Diastolic Blood Pressure } & $75.92 \pm 10.30$ & $74.21 \pm 10.81$ & - & $0.340^{c}$ \\
\hline
\end{tabular}


Table 4 Predictors of the risk factors of Diabetes among the Tuberculosis Patients

\begin{tabular}{|c|c|c|}
\hline Characteristics & Crude Odds Ratio (95\% Cl) & Adjusted Odds Ratio (95\% Cl) \# \\
\hline \multicolumn{3}{|l|}{ Family History } \\
\hline Yes & $5.095(2.500-10.382)^{*}$ & $2.463(0.638-10.024)$ \\
\hline No & Reference & Reference \\
\hline \multicolumn{3}{|c|}{ Current Use of Tobacco Products } \\
\hline Yes & $3.155(1.515-6.570)^{*}$ & $2.247(0.728-11.984)$ \\
\hline No & Reference & Reference \\
\hline \multicolumn{3}{|c|}{ Current Use of Alcohol (within 30 days) } \\
\hline Yes & $16.167(4.262-61.322)^{*}$ & $12.307(2.856-57.067)^{*}$ \\
\hline No & Reference & Reference \\
\hline \multicolumn{3}{|l|}{ Stress Level } \\
\hline Normal & Reference & Reference \\
\hline Mild-Moderate & $0.373(0.139-0.999)^{*}$ & $1.930(0.407-9.158)$ \\
\hline Severe & $0.823(0.269-2.514)$ & $3.816(0.540-26.983)$ \\
\hline
\end{tabular}

"** Significant Association $(P<0.05)$,

"\#" Adjusted for current tobacco user, current alcohol drinker, family history, and stress

Despite, the low prevalence of diabetes (11.9\%) in our study, there still exist a considerable portion (17.8\%) of the respondents with impaired glucose and impaired fasting (17.8\%). These findings are slightly lower than the study done in Tamil Nadu, India where diabetes and pre-diabetes were 25.3 and $24.5 \%$, respectively [8]. However, the considerable portion of respondents with a family history of diabetes in both the studies; Tamil Nadu and our study justifies the pre-diabetic cases.

On the contrary to the study done in Kerala, India [9] which reported $55.6 \%$ of sputum positive cases, the present study reported only $39.4 \%$ of the sputum positive cases. This is somehow aligned by the fact that the present study included only the respondents who had completed the intensive phase ( 2 months) of the tuberculosis treatment regimen, unlike the study done in Kerala, India which had all the new and old cases under the tuberculosis treatment.

\section{Behavioral characteristics}

Among the current user of tobacco products, many respondents 35 (60.3\%) preferred smokeless tobacco which is comparatively higher (11.7\%) than the study conducted in Tamil Nadu [8]. Tobacco product contains nicotine which has established evidence in insulin secretion and action, hence it justifies the association of the current user and non-user of tobacco products with diabetes-tuberculosis comorbidity [10].

Likewise, harmful use of alcohol is considered as the potential risk for onset of diabetes along with other chronic diseases by causing insulin resistance and pancreatic B-cell dysfunction [11].

However, the study findings reflect a small portion of $14(4.3 \%)$ of the respondents who currently drinks the alcohol correlates the findings from the study done in Nepal in 2013 [4].

One of the irreversible risk factors of diabetes is family history, and as the findings from the study done in Puducherry [7] suggests, the odds of acquiring diabetes is 4.096 times (OR $=4.096,1.730-9.698)$. This finding is higher, yet remains coherent in our study as well where the family history is positively associated with diabetes, and the odds of getting diabetes is 2.463 times $(\mathrm{OR}=$ $2.463,0.638-10.024)$.

\section{Physical measures}

Unlike Body Mass Index (BMI), waist-to-hip ratio is considered as the better predictor of abdominal obesity which correlates with the metabolic syndrome and other diseases [12]. The study reported collectively, $40.0 \%$ of the total respondents to have abdominal obesity which is higher than the findings from other studies done in Nepal [4] and India [8] where the abdominal obesity range from 5.2 to $34.9 \%$.

With regard to the blood pressure of the respondents, the high blood pressure and diabetes have substantial overlap and frequently occurs simultaneously. Hypertension and diabetes share a common pathway and risk factors like obesity, physical inactivity, and unhealthy lifestyle.

\section{Stress level}

Stress is associated with the release of cortisol, responsible for "flight and fight" response leading to a hyperglycemic state of the body. However, prolonged stress is a potential contributor to diabetes [13]. 


\section{Conclusion}

To conclude, for every known case of diabetes, there are as many cases with impaired glucose tolerance and prediabetic, hence it may be inferred that active tuberculosis may be an alerting sign to unmask those pre-diabetic case for early diagnosis and treatment; shifting the focus on the bidirectional screening of tuberculosis and diabetes.

\section{Recommendation}

As the study findings suggest current alcohol consumer as a significant predictor of Diabetes among the Tuberculosis patient on treatment, there exist the need to prioritize these populations in the adoption of bidirectional screening program for TB and DM. Furthermore, a prospective study, with the addition of the comparator group as general population would have strengthened the DM-TB comorbidity. Additionally, analyzing the blood sample by same trained laboratory personal would eliminate the inter-observer bias.

\section{Abbreviations}

DM: Diabetes Mellitus; DOTS: Direct Observed Treatment Short Course; HIV: Human Immune Deficiency Virus; LMIC: Low and Middle Income Country; MDR-TB: Multidrug-Resistant Tuberculosis; NCD: Non-Communicable Disease; SEAR: South-East Asian Region; TB: Tuberculosis; TB-DM: Tuberculosis Diabetes comorbidity; WHO: World Health Organization

\section{Acknowledgments}

Author would like to express sincere gratitude to Dr. Vijay Kumar Khanal, Dr. Nilambar Jha, Dr. Prajiwal Pyakurel, Mrs. Gyanu Nepal, Dr. Shyam Sundar Budathoki, Mr. Bishal Sharma, Mr. Sanjeeb Shah, to all the staffs of Health Office, Health Post, Primary Health Care Center, Hospital and health centers of Morang and also to all the participants of the study.

\section{Authors' contributions}

Each author and co-authors have made a substantial contribution to the study. However, the design and acquisition were mainly contributed by the co-authors; VKK, NJ, PP, GN whilst, the corresponding author BS, considerably contributed regarding the data collection, analysis, and interpretation. All the authors read and approved the final manuscript and also have agreed related to the accuracy and integrity of the work.

\section{Funding}

Not applicable.

\section{Availability of data and materials}

The datasets generated and/or analyzed during the current study are not publicly available due to privacy policy of the human subject involved in the study but can be made available from the corresponding author on reasonable request.

\section{Ethics approval and consent to participate}

Approval for this study was obtained following proposal review from Institutional Review Committee (IRC) of BPKIHS (Code No: IRC/1265/018). Informed written consent was taken from respondents.

\section{Consent for publication}

Not applicable

\section{Competing interests}

Not applicable.
Received: 25 April 2019 Accepted: 1 November 2019

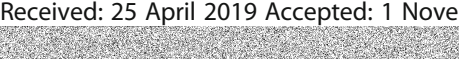

\section{References}

1. World Health Organization. Diabetes [Internet]. 2018 [cited 2019 Feb 24]. Available from: https:/www.who.int/en/news-room/fact-sheets/detail/diabetes.

2. Lin Y, Li L, Mi F, Du J, Dong Y. Li Z, et al. Screening patients with Diabetes Mellitus for Tuberculosis in China. 2012;00(00):1-7.

3. Manuscript A. epidemics. 2010;9(12):737-46.

4. Thapa B, Paudel R, Thapa P, Shrestha A, Ak P. Prevalence Of Diabetes Among Tuberculosis Patients And Associated Risk Factors In Kathmandu Valley. :20-7.

5. Diabetes Mellitus and Associated Risk Factors among Tuberculosis Patients in Rawalpindi. 2017;1(4):1-6.

6. Wang $Q, M a A, \operatorname{Han} X$, Zhao S, Cai J, Ma Y, et al. Prevalence of Type 2 Diabetes among Newly Detected Pulmonary Tuberculosis Patients in China : A Community Based Cohort Study. 2013;8(12):1-8.

7. Raghuraman S, P KV, Govindarajan S, Chinnakali P, Panigrahi KC. Prevalence of Diabetes Mellitus among Tuberculosis Patients in Urban Puducherry. 2014;6(1).

8. Viswanathan V, Kumpatla S, Aravindalochanan V, Rajan R. Prevalence of Diabetes and Pre-Diabetes and Associated Risk Factors among Tuberculosis Patients in India. 2015;7(7):1-9.

9. Mrithyunjayan S, Wilson N, Satyanarayana S. Dewan PK. High Diabetes Prevalence among Tuberculosis Cases in. 2012;7(10):1-7.

10. Xie X, Liu Q, Wu J, Wakui M. Impact of cigarette smoking in type 2 diabetes development. 2009;30(6):784-7.

11. Kim S, Kim D. Alcoholism and Diabetes Mellitus. 2012;108-15.

12. Very well Health. BMI, Waist Circumference, or Waist-To-Hip Ratio? [Internet]. 2017 [cited 2019 Apr 24]. Available from: https:/www.verywellhealth.com/ bmi-waist-circumference-waist-to-hip-ratio-1745981.

13. Surwit RS, Schneider MS, Feinglos MN. Stress and diabetes mellitus. Diabetes Care [Internet]. 1992t [cited 2019 Apr 24];15(10):1413-22. Available from: http://www.ncbi.nlm.nih.gov/pubmed/1425110.

14. World Health Organization. WHO STEPS Instrument (Core and Expanded) STEPS Instrument Overview. 2012;18. Available from: www.who.int/chp/steps.

15. Smith D. Stress Assessment questionnaire. (0):20-1. Available from: https:// maic.qld.gov.au/wp-content/uploads/2016/07/DASS-21.pdf.

\section{Publisher's Note}

Springer Nature remains neutral with regard to jurisdictional claims in published maps and institutional affiliations.
Ready to submit your research? Choose BMC and benefit from:
- fast, convenient online submission
- thorough peer review by experienced researchers in your field
- rapid publication on acceptance
- support for research data, including large and complex data types
- gold Open Access which fosters wider collaboration and increased citations
- maximum visibility for your research: over $100 \mathrm{M}$ website views per year
At BMC, research is always in progress.
Learn more biomedcentral.com/submissions 